\title{
The Influential Articles and Topics in the Journal of Korean Neurosurgical Society Publications between 2019 and 2020
}

\author{
Hee-Jin Yang, ${ }^{1}$ Chul-Kee Park, ${ }^{2}$ Moonyoung Chung ${ }^{3}$ \\ Editor in Chief, Journal of Korean Neurosurgical Society; Department of Neurosurgery, Seoul National University Boramae Hospital, Seoul, \\ Korea \\ Associate Editor, Journal of Korean Neurosurgical Society; Department of Neurosurgery, Seoul National University Hospital, Seoul National \\ University College of Medicine, Seoul, Korea \\ Managing Editor, Journal of Korean Neurosurgical Society; Department of Neurosurgery, Soonchunhyang University Bucheon Hospital, \\ Soonchunhayng University College of Medicine, Bucheon, Korea
}

The 2020 impact factor (IF) of the Journal of Korean Neurosurgical Society (JKNS) was 1.729 , an increase of $26 \%$ from the previous year (1.376). The rise in IF and sustaining its upward trend in recent years is very encouraging ${ }^{4)}$. It is the results of the unremitting efforts of all members of the Korean Neurosurgical Society (KNS). Awareness of the importance of journal and support from KNS were also of great help ${ }^{3,4}$. In this regard, it is meaningful to discover the most cited papers, topics, and fields published in JKNS.

\section{THE INFLUENTIAL ARTICLES}

The citation data was extracted from the Web op Science on November 28, 2021. The number of articles published in JKNS between January 2019 and November 2020 was 181. The total number of citations was 524, and the average number of citations per article during that period was 2.89. As many as 137 articles were cited at least once, accounting for $75.7 \%$ of the total.

Interestingly, compared to the data published in 2020, the average citation number per article increased in accordance with the increase in journal impact factor (JIF), whereas the proportion of uncited articles did not decrease significantly $(24.3 \% \text { vs. } 24.5 \%)^{2}$. This means that the proportion of more influential articles has increased. A quarter of all citations (131/524) came from 10 out of 181 articles (5.5\% of all published articles), indicating the importance of influential articles.

Among the frequently cited articles, six influential articles that received the highest international attention and contributed greatly to the reputation of the journal were selected. It is our honor to announce these articles with the list of the corresponding authors (Fig. 1).

Jung et al. Factors Related to Successful Energy Transmission of Focused Ultrasound through a Skull : A Study in Hu-

- Received : December 27, 2021 •Revised : December 29, 2021 •Accepted : December 29, 2021

- Address for reprints : Hee-Jin Yang

Department of Neurosurgery, Seoul National University Boramae Hospital, 20 Boramae-ro 5-gil, Dongjak-gu, Seoul 07061, Korea

Tel : +82-2-870-2303, Fax : +82-2-870-3863, E-mail : nsyangdr@gmail.com, ORCID : http://orcid.org/0000-0002-6413-1363

This is an Open Access article distributed under the terms of the Creative Commons Attribution Non-Commercial License (http://creativecommons.org/licenses/by-nc/4.0) which permits unrestricted non-commercial use, distribution, and reproduction in any medium, provided the original work is properly cited. 
man Cadavers and Its Comparison with Clinical Experiences. 2019;62(6):712-722 by Jin Woo Chang of Yonsei University College of Medicine.

Kim et al. Obtaining Informed Consent Using Patient Specific 3D Printing Cerebral Aneurysm Model. 2019;62(4):398404 by Jae IL Lee of Pusan National University Hospital.

Yang et al. The Present and Future of Vagus Nerve Stimulation. 2019;62(3):344-352 by Ji Hoon Phi of Seoul National University Children's Hospital.

Chang et al. Human Induced Pluripotent Stem Cells : Clinical Significance and Applications in Neurologic Diseases. 2019;62(5):493-501 by Sang-Dae Kim of Korea University Ansan Hospital.

Ceren et al. Comparison of Biomechanical Properties of Dura Mater Substitutes and Cranial Human Dura Mater : An In Vitro Study. 2019;62(6):635-642 by Ceren Kizmazoglu of Dokuz Eylul University School of Medicine

Ko et al. Correction of Spondylolisthesis by Lateral Lumbar Interbody Fusion Compared with Transforaminal Lumbar Interbody Fusion at L4-5. 2019;62(4):422-431 by Seung Won Park of Chung-Ang University Hospital

Looking at the types of selected articles, there were three clinical studies, two reviews, and one laboratory investigation. As usual, this means that recruiting review articles will help improve the reputation of JKNS. However, the original articles (clinical study and laboratory investigation) published in JKNS also show that they make a significant contribution as
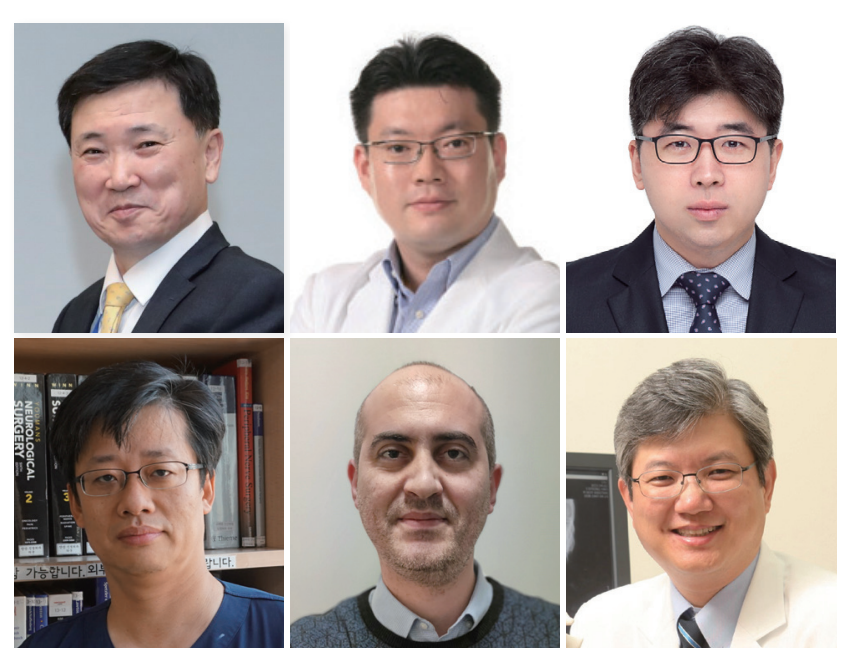

Fig. 1. The corresponding authors of the six influential articles in the Journal of Korean Neurosurgical Society publication between the years 2019 to 2020. From top left to bottom right: Jin Woo Chang, Jae II Lee, Ji Hoon Phi, Sang-Dae Kim, Ceren Kizmazoglu, and Seung Won Park. much as reviews, showing a good balance in quality in both the requested review articles and voluntarily submitted original articles. It is well known that most academic journals have about two thirds or three quarters of articles with fewer citations than $\mathrm{JIF}^{1)}$. This trend is also found in JKNS, which indicates that the value of a scientific journal depends on whether they publish more articles that have higher academic significance.

\section{THE FIELDS AND TOPICS OF ARTICLES WHICH GOT MORE CITATIONS}

The selected influential articles covers most of the major fields of neurosurgery, which are functional, cerebrovascular, spine, pediatric and basic research. However, there is relative paucity of articles in neuro-oncology field among the frequently cited articles when compared to other clinical fields of neurosurgery. Considering that Korean researchers are making many achievements in the field of brain tumors, it will be of great help to the development of JKNS if domestic researchers are motivated to publish many brain tumor-related articles. It is also notable that case reports are not listed in the frequently cited articles. It is thought that the editorial board should control the number of published case reports by applying stricter standards concerning the probability of citation in mind when reviewing submitted case reports. Articles on 'focused ultrasound' is one of the influential articles, and its originality was its strong point as an influential article. The goal of KNS members is to develop novel topics of interest to overseas readers. Other interesting topics are emerging fields such as artificial intelligence and $3 \mathrm{D}$ printing. This suggests that selecting a 'hot topic' for review papers will be a great help in increasing JKNS's citations. In keeping with the rapid development of academia and changing trends, the editorial board should always pay attention to the fields that are receiving attention.

\section{CONFLICTS OF INTEREST}

No potential conflict of interest relevant to this article was reported. 


\section{AUTHOR CONTRIBUTIONS}

Conceptualization : HJY, CKP, MC

Data curation : $\mathrm{MC}$

Formal analysis : CKP

Funding acquisition : HJY

Methodology : HJY

Project administration : HJY

Visualization : HJY, CKP, MC

Writing - original draft : HJY, CKP, CM

Writing - review \& editing : HJY, CKP, CM

\section{References}

1. Diamandis EP : The Journal Impact Factor is under attack - use the CAPCl factor instead. BMC Med 15 : 9, 2017

2. Im SB, Chung M, Kim BT : The influential articles and title words in the Journal of Korean Neurological Society publications between the years 2016 to 2018. J Korean Neurosurg Soc 63 : 1-3, 2020

3. Yang HJ : Journal of Korean Neurosurgical Society from 2001 to 2006, the pivotal period of transition and preparation for making great stride. J Korean Neurosurg Soc 62 : 131-135, 2019

4. Yang HJ, Park CK, Chung M : Journal citation report 2020 and impact factor of Journal of Korean Neurosurgical Society. J Korean Neurosurg Soc 64 : 675-676, 2021

\section{ORCID}
Moonyoung Chung https://orcid.org/0000-0003-4733-6321
Chul-Kee Park
https://orcid.org/0000-0002-2350-9876
Hee-Jin Yang
https://orcid.org/0000-0002-6413-1363 